eCommons@AKU

March 2016

\title{
Iron Deficiency Anemia among Women of Reproductive Age, an Important Public Health Problem: Situation Analysis
}

\author{
Minaz Mawani \\ Aga Khan University, minaz.mawani@aku.edu \\ Savera Aziz Ali \\ Aga Khan University, savera.azizali@aku.edu \\ Gulshan Bano \\ Aga Khan University, gulshan.bano@aku.edu \\ Sumera Aziz Ali \\ Aga Khan University, sumera.ali@aku.edu
}

Follow this and additional works at: http://ecommons.aku.edu/

pakistan_fhs_mc_med_intern_med

Part of the Internal Medicine Commons, Obstetrics and Gynecology Commons, and the Preventive Medicine Commons

\section{Recommended Citation}

Mawani, M., Ali, S. A., Bano, G., Ali, S. A. (2016). Iron Deficiency Anemia among Women of Reproductive Age, an Important Public Health Problem: Situation Analysis. Reproductive System \& Sexual Disorders: Current Research., 5(3), 1-6.

Available at: http://ecommons.aku.edu/pakistan_fhs_mc_med_intern_med/32 


\title{
Reproductive System \& Sexual

\section{Iron Deficiency Anemia among Women of Reproductive Age, an Important Public Health Problem: Situation Analysis}

\author{
Minaz Mawani ${ }^{1}$, Savera Aziz $\mathrm{Ali}^{2}$, Gulshan Bano ${ }^{3}$ and Sumera Aziz $\mathrm{Ali}^{3}$ \\ ${ }^{1}$ Department of Medicine, Aga Khan University Hospital Karachi, Pakistan \\ ${ }^{2}$ School of Nursing and Midwifery Aga Khan Hospital Karachi, Pakistan \\ ${ }^{3}$ Department of Community Health Sciences, Aga Khan University Hospital Karachi, Pakistan
}

*Corresponding author: Ali SA, Department of Community Health Sciences, Aga Khan University Hospital Karachi, Pakistan, Tel: +92-21-34930051; E-mail: sumera.ali@aku.edu

Rec date: July 13, 2016; Acc date: August 3, 2016; Pub date: August 9, 2016

Copyright: () 2016 Mawani M, et al. This is an open-access article distributed under the terms of the Creative Commons Attribution License, which permits unrestricted use, distribution, and reproduction in any medium, provided the original author and source are credited.

\section{Abstract}

Background: Iron deficiency is a major cause of anemia and is more prevalent in developing countries, posing additional burden on health care systems, in the presence of scarcity of resources. The high-risk group consists of females and children but comparatively it is higher in females due to physiological reasons. Multiple factors can predict anemia, which in turn leads to various adverse outcomes.

Objective: The purpose of this literature review was to review and synthesize the findings from literature about the burden, causes, outcomes and important interventions of iron deficiency anemia for developing countries.

Methods: An extensive literature review was carried out by retrieving articles from various databases like Pub med, Google scholar, Science direct, World Bank and WHO databases. Relevant articles were retrieved from different databases by using following text words and phrases: 'anemia', 'Iron deficiency', 'risk factors of anemia', 'outcomes of anemia', 'interventions' and developing countries.

Results: Multiple determinants of iron deficiency anemia have been identified in the literature including genetic and environmental factors. Some of these factors are lack of proper diet, poor socioeconomic status, high parity of women, access to poor health and genetic etc. Moreover, anemia can result in various poor outcomes like fatigue, poor mental health, lack of concentration, poor feto-neonatal outcomes like stillbirth, preterm birth and postpartum Hemorrhage.

Conclusion: Iron deficiency anemia is one of the important public health problems in developing countries among the women of reproductive age group. Multiple factors like age, parity, socioeconomic status and diet can determine the stores of iron in the women of reproductive age group. Anemia can lead to multiple adverse outcomes, which can be prevented by doing appropriate cost-effective interventions on time.

Keywords: Iron deficiency; Women; Reproductive age; Developing countries; Literature review

\section{Introduction}

Iron is one of the minerals required by our body to carry out some of the essential functions. The basic and important function of iron (as a part of hemoglobin) is to transport oxygen from lungs to cells in our body and is an essential requirement of the body.

According to $\mathrm{CDC}$ (centers for disease control and prevention), "Iron deficiency is a condition resulting from too little iron in the body".

Iron is important for the production of hemoglobin by erythroblasts. If iron supply is not adequate, the HB production does not succeed and the number of red blood cells decreases [1]. This condition is also known as "Anemia".

Anemia is detected by a hemoglobin concentration less than a certain recommended level. According to WHO the recommended
Hemoglobin level, for non-pregnant women (age 15 and over) is $120 \mathrm{gm} / \mathrm{L}$ and for males (15 years and over) it is $130 \mathrm{gm} / \mathrm{L}[1]$.

Furthermore, iron deficiency can be categorized into three categories;

- Mild Deficiency consists of depleted stores with normal production of $\mathrm{Hb}$ and iron- dependent protein.

- Marginal deficiency consists of depleted iron stores and decreased iron-dependent protein production but normal $\mathrm{Hb}$ concentrations.

- Iron deficiency anemia consists of decreased concentration of circulating RBCs which results in decreased concentration of $\mathrm{Hb}$ within blood cells resulting in compromised transport of oxygen to tissues, iron stores are further depleted and the concentrations of iron-dependent oxidative enzymes are reduced [2].

Anemia has been a major public health concern over the past few years. According to $\mathrm{N}$ Milman, "The most common nutrition deficiency in both developing as well as in developed countries is iron deficiency" [1]. Globally, similar to other diseases, poor countries and underprivileged group suffer the most. 
In poorest countries, pregnant women and children are the most anemic population group and have the least access to interventions and services. According to a report by World Bank on anemia, "In women, anemia prevalence decreases with income in every region/ country". Prevalence in the poorest as compared to richest is twice high in many countries [3]. A study was carried out in Abbottabad and the results showed that the most common type of anemia is an iron deficiency anemia that affects $68 \%$ people and is more common in females [4].

Although iron deficiency is common among women of developing as well as industrialized countries, statistics show that it is more common in developing countries [5]. Multiple causes and consequences of anemia in developing countries have been reported in the literature and these are not synthesized at one place.

Thus the purpose of this literature review was to review and synthesize the findings from literature about the burden, causes, outcomes and important interventions of anemia for developing countries.

\section{Methods}

\section{Search strategies and data sources}

An extensive literature review was carried out by retrieving articles from various databases like Pub med, Google scholar, Science direct, World Bank and WHO databases. In addition to this; different reports were also being studied including World Health Organization and World Bank reports and Pakistan Demographic and Health Survey 2013, Demographic health survey (DHS) reports, UNICEF: statistics. Relevant articles were retrieved from different databases by using following text words and phrases: 'Anemia', 'Iron deficiency', 'risk factors of anemia', 'outcomes of anemia', 'interventions' and 'developing countries'.

\section{Eligibility criteria}

Types of studies included were descriptive studies, observational studies, correlational studies and comparative studies. Complete papers were reviewed and included rather than the abstracts only. Electronic search was carried out for the papers, which were published in last fifteen years in both in developed and developing countries. All the studies which had been written in the English language, reporting data on iron deficiency anemia, determinants, outcomes and interventions of anemia were included in this review.

\section{Data abstraction}

Data was abstracted from all of the included studies. Abstracted data included study design; type of database used for analysis (population-based or hospital-based) if any; characteristics of the study subjects, definition of Anemia; potential confounders or effect modifiers considered.

\section{Findings of the Literature Review}

Iron deficiency is a major cause of anemia and is more prevalent in developing countries, posing additional burden in their health care systems, when already there is a major lack of resources. The high-risk group consists of females and children but comparatively it is higher in females due to physiological reasons. Presence of some social factors further aggravates the problem. Despite several interventional programs already in working conditions, its prevalence is still very high. This poses for additional analysis of interventions and possibilities. The prevalence of anemia in developing countries is three to four times higher than in industrialized countries. The most highly affected population groups in developing countries are pregnant women (56\%), school age children (53\%), non-pregnant women (44\%), and preschool children (42\%) [6].

\section{Global situation analysis}

"Regardless of its etiology, the World Health Organization (WHO) estimates that anemia affects over 1.62 billion people worldwide. The most affected group is preschool-age children, with a prevalence of $47 \%$, followed by pregnant women $(41 \%)$, non-pregnant women (30\%), school-age children (25\%), and people older than 60 years of age $(24 \%)$; men are the least affected group (12\%). However, globally, the most numerous population group is non-pregnant women (468.4 million)" [7].

According to WHO, most affected regions are Southeast Asia and Africa [8]. Whereas the least affected regions are western pacific and Europe [9].

On an average, globally, about $50 \%$ of the anemia is assumed to be attributable to iron deficiency. Additionally, Iron deficiency ranks number 9 among 26 risk factors included in the GBD (Global burden of disease project, WHO 2000), and is a reason for 841,000 deaths and $35,057,000$ disability-adjusted life years lost. $71 \%$ of the global mortality burden and $65 \%$ of the disability-adjusted life years lost are contributed by Africa and parts of Asia, whereas North America bears $1.4 \%$ of the global burden [10].

"For women and young children, the majority of WHO Member States (132 to 159 , depending on the population group) have a moderate-to-severe public health burden of anemia; meaning that over $20 \%$ of the population group in these countries is affected" [8].

Although interventions are already in effect but the prevalence is still high. These statistics show that this problem needs to be relooked into and interventions redesigned according to population need. In all the countries globally, the prevalence of anemia has a "social imbalance". It is more prevalent in lower socioeconomic strata and population with low educational status [1].

The prevalence of anemia in developed countries particularly 48 hours after delivery is approximately $50 \%$ whereas in developing countries, it is in the range of $50-80 \%$ [11].

\section{Local situation analysis}

Anemia is most prevalent in Asia. About half of all the anemic women live in the Indian subcontinent where $88 \%$ of them develop anemia during pregnancy [6]. It is associated with poor perinatal outcomes, especially in developing countries. Several of the studies have added to the outcome including one done at AKUH in 2001-2002 [12].

Recent statistics shows a prevalence of around $45 \%$ of Iron deficiency anemia in Pakistan, which is quite high realizing the failure of public health measures to control it.

According to EMRO WHO statistics, it has been found that more than one-fifth of women in Pakistan suffer from anemia. At the 
country level, awareness exists regarding this lasting health problem in the country as verified by several regional surveys [6].

A cohort study was done in an urban setting in Hyderabad from 2003-2005, where pregnant women were studied. It revealed that the prevalence of iron deficiency anemia was $90.5 \%$ out of which $75 \%$ had mild anemia, $14.8 \%$ had moderate anemia and $0.7 \%$ were severely anemic. The causes identified were primarily nutrition related. Pica, tea consumption and lesser intake of hem-iron sources were leading causes [13].

In Pakistan, the prevalence of anemia among ever-married women aged 15 to 44 is reported to be $26 \%$ in urban areas and $47 \%$ in rural Areas. The prevalence of anemia among pregnant women living in urban areas is similar, ranging from $29 \%$ to $50 \%$ [13].

Another study done in Ayub teaching hospital consisting of 100 anemic patients, revealed that iron deficiency anemia is the most common form of anemia in Pakistan. It is more common in females and in the age group 21-60 years, with two peaks, one in 21-30 years and the other in 41-50 years [4].

\section{Causes of iron deficiency}

Causes of iron deficiency fall into two broad categories. One is increased iron needs and another is decreased iron intake/absorption [1].

Increased iron needs may be due to the increasing needs of the body as part of development, blood loss, worms, pregnancy, infections, inflammatory bowel disease or donations. Furthermore, women with gynecological diseases or having heavy blood losses at menstrual periods are at an additional higher risk of developing anemia. Genetic disorders like thalassemia and sickle cell disease are also associated with and are aggravated by the presence of anemia [1]. Additionally, bone marrow diseases that cause suppression of red cells synthesis, chronic renal failure, rheumatoid arthritis and tuberculosis are also some of the causes that lead to anemia [4].

During the whole time period of pregnancy, there is an increased need of about 700-850 mg of body iron while lactation results in additional loss of iron via breast milk. But, lactation amenorrhea compensates for this loss [6]. The problem increases with repeated pregnancies as body iron stores get depleted. Among preschool children, the magnitude of anemia is reported to be associated with birth order thereby indicating a gradual depletion of the iron stores of mothers after repeated pregnancies [6].

Decreased absorption may be due to effects of some medications that hinder the absorption of iron from the diet. Another possibility is a lesser intake of dietary sources of iron. The average daily dietary iron intake in developed countries is between 10 and $15 \mathrm{mg}$. under normal conditions, only between $5 \%$ and $10 \%$ of this quantity is absorbed, that is, $1-3 \mathrm{mg} /$ day [9]. This relates to improving iron intake. Prevalence of anemia is observed to be high in large families and those in which mothers have lower literacy levels. Cultural beliefs also have a large impact on it. Some cultural groups avoid iron- rich sources of diet and that makes a large proportion of world's population [6].

Obesity can be another factor of iron deficiency. Studies done in transitional countries have indicated that obesity increases the risk of iron deficiency due to adiposity-related decreased dietary absorption. Cross-sectional studies in industrialized countries have shown that obese individuals are at increased risk of iron deficiency [14].
In developing countries most common cause of anemia is malnutrition and poor dietary intake [3,9]. Most women from developing and developed countries consume a diet lacking in haemiron and consume a diet adequate in non-haem iron, that has a low absorption [1].

\section{Outcomes of anemia}

The reason why Iron deficiency is a matter of concern is that it causes fatigue, decreased motor abilities, impaired memory and can also cause delays in mental development $[15,16]$. In addition to this, anemia in pregnancy can increase the risk of having a preterm delivery and preterm babies have other health concerns ranging from low birth weight and neural tube defects to death. A preterm birth is one of the main causes of infant mortality in developing countries and iron supplementation during pregnancy helps in reduction of risk of preterm birth [9].

The trend in developing countries is that adolescent girls achieve menarche being iron deficient and since it is not treated with diet due to lack of resources or awareness, it is carried on till pregnancy and leads to natal and post natal complications. "Infants and children born to iron-deficient mothers have a poorer cognitive development of the brain functions and a lower intelligence quotient than infants and children born to iron-replete mothers" [1]. The deficiency is carried on with multiple pregnancies and hence compromises health and increases risk of complications.

Common sign and symptoms of anemia include feeling lethargy, slow development, difficulty in maintaining body temperature, decreased immune function, inflamed tongue headache, palpitations, dizziness, emotional instability, depression and restlessness [1]. One of the studies also indicates the increased occurrence of stress fractures related to anemia [15].

\section{Intervention options to address the problem}

Dietary modifications: Interventions range from primary prevention that involves a focus on a healthy diet and including sources of iron. Good sources of iron include fruits, vegetables, whole grains, milk and milk products, lean meat, fish, dry beans, egg, nuts. A healthy diet includes diet low in saturated fats, trans fats, cholesterol, sugars and salt. Diet that can help in absorption of iron includes good sources of vitamin C (appendix III) and non-haem iron. This can especially be more effective when taken at the same time. Sources of non-haem iron include soy beans, kidney beans, chickpeas tomato puree etc. Different interventions are available for different age groups. Babies should be breastfed for at least a year and when the diet is started, it should contain iron-rich cereal or pureed meat. The interventions should start from earlier in childhood so that children grow healthy and not become deficient in the later part of their life. Fruits, vegetables and other sources of vitamin $\mathrm{C}$ should be added to the diet to meet the needs of growing children. Groups like young children, adolescent girls, women of reproductive age, pregnant and lactating women should be an area of focus for interventions [1]. Iron levels can be detected via blood works of Hemoglobin, Hematocrit, $\mathrm{CBC}$, serum iron, ferritin, transferrin saturation and transferrin receptor.

Iron supplements: Treatment for iron deficiency depends on age, health and specific cause of the deficiency. If the cause is related to dietary intake then increasing dietary sources of iron can help. Additionally, iron supplements are also prescribed in different settings. 
Besides this, cases are followed at intervals for blood levels. There are several success stories of countries implementing interventions and getting a good outcome. One of them is of Thailand. During the year 1986 to 1996/7 Thailand was successful in decreasing anemia in pregnant women with the help of 500,000 trained volunteers to identify women in their early pregnancies and encouraging the use of antenatal services. They were provided with iron supplements and education on usage and importance. In addition, they were also treated for malaria and worms [3]. WHO recommends that in high-risk populations, iron supplements should be given to adolescents and women for 2 to 4 months per year to ensure that women have reasonable iron stores when they become pregnant [13].

In all the countries, iron needs in pregnancy should be considered for individual females and iron supplementations should be prescribed according to the need. In developing countries, it is easy to identify and treat cases of iron deficiency since access to health care is good [1]. It is important to check for the levels of hemoglobin 2-4 weeks after initial doses. It should be continued for at least 6 months after $\mathrm{Hb}$ concentrations have reached to normal levels in order to build iron stores. Intravenous iron should be considered in levels less than $90 \mathrm{~g} / \mathrm{L}$.

Antihelminthic treatment: Interventions besides diet and supplements include the use of anthelminthics in at-risk population. However there is a lack of evidence regarding its effectiveness and thus cannot be recommended in out setting [16].

Food fortification: Promotion of foods rich in iron as well as micronutrients (folic acid, Vitamin A, C, B-12, and Zinc) help in iron absorption. Iron fortification is one of the strategies that was implemented in the west but later on it was brought to an end. The reason was that in the western countries iron overload (hemochromatosis) in male population was already prevalent so introducing iron-fortified diet for general population may prove risky for a part of the population. People with this kind of disorder have already high intestinal iron absorption. In the western countries, the focus should be on specific groups for iron supplementation rather than on general population which may lead to iron overload and have harmful effects [1].

A recent advancement is the development of a variety of "in-home fortificants". These range from small packets of encapsulated micronutrients that can be mixed with any complementary food. Among these, the sprinkles developed at sick kids hospital in Toronto Canada is now being used in several countries for infants and young children, and as part of the emergency relief effort in Indonesia following the December 2004 tsunami [6]. In order to enhance the absorption of iron, vitamin B-12 is recommended along with others. Rich sources of Vitamin B-12 are meat and poultry [1].

Managing post-partum complications and family planning: "The average menstrual blood loss is $35 \mathrm{ml} /$ cycle, with a range of $25-60 \mathrm{ml}$. It is lower in women using oral contraception and higher in women using intrauterine contraceptive device" [2]. Postpartum anemia is a problem that needs to be worked upon. Additionally, those methods of family planning should be used that decrease bleeding [2]. Birth spacing is also very important as it helps in replenishing depleted iron stores as a result of pregnancy and childbirth [17].

Other interventions: Other interventions include control of malaria and other infectious diseases [18]. Interventions on public level include analysis of the problem, raising awareness, building partnerships within different sectors like health, agriculture, pharmaceuticals, nongovernmental organizations and government agencies. Review of existing programs and their impact is highly essential for improvement. Monitoring programs should be developed. Since in our settings women do not have access to health care resources so it is important to identify and treat cases of iron deficiencies, malaria, helminths and focus on high-risk groups (antenatal, delivery and postpartum). Besides, use of behavior change strategies can also be useful.

\section{Applicability to Pakistani population}

In developing countries, dietary intake of iron is already of substandard level due to lack of availability and affordability. The majority of diet content either consists of plant origin or of non-haem iron sources which have a low bioavailability. The strong inhibitors of iron absorption like cereal grains and tea are also a part of the routine diet. Nutritional measures should be taken in high-risk groups especially, but the issue of applicability remains a question in the presence of low socioeconomic status and especially poor countries [1].

Iron fortification of food can be an appropriate measure since iron overload is not common and at least $90 \%$ of the female population have anemia [1], but in areas where infectious diseases are endemic, this would not be as applicable since malaria may be aggravated by the presence of plenty of iron stores. Although studies do not show any evidenced change in the status of malaria in iron supplemented children [1]. In Southeast Asian countries "NaFeEDTA" fortified fish sauce is seen to improve iron status. Several Southeast Asian countries are planning to introduce iron -fortified flour [1].

Use of iron and multivitamin sprinkles are also effective. It can easily be added to the routine diet. A randomized controlled trial was done in India in 2004, it was concluded that "sprinkles" were very effective in treating iron deficiency [19]. Its applicability in Pakistani population can be suggested since it has already been tried in a developing country. In regions of higher prevalence of anemia, iron supplementations should be used frequently to cover high- risk groups [1].

Some studies suggest that iron and folic acid supplementation should be prescribed when a woman decides for pregnancy to make sure that adequate iron reserves are present during pregnancy and postpartum period. This is especially true in the areas of high prevalence of iron deficiency e.g. Southeast Asia and Africa. It is also suggested that in areas of high prevalence doses of iron supplementation should be higher than in general population [1] There are studies from Indian subcontinent that demonstrate that intravenous iron therapy can be very efficient in the correction of iron deficiency anemia in late pregnancy. It is convenient for the patient and reduces overall health care cost [1].

In contrast to the situation in western countries, there is a lack of facilities and access to health care in developing countries. There are no facilities for assessment of ferritin levels and there is a need to do that since the prevalence of anemia is quite high. It should be made available on field levels [1].

Vitamin B-12 is also one of the essential vitamins. Its sources are meat and poultry so the issue of affordability arises in developing countries [1]. The Government should focus on subsidizing rates for these sources in order to decrease the additional burden on health care resources as well as decrease morbidity and mortality. Combine tablets that contain iron and micronutrient together will be more convenient 
since it does not require ingesting separate pills for each component [1].

Frequent follow-ups and reminders would be beneficial. The door to door approach should be implemented, keeping in mind the cultural implications and lack of access to medical facilities for women. Counseling should involve all family members, considering men as well that would help them to accept the idea. Pakistan has a male dominating society; hence male member of the family should be made aware of this problem. Sometimes when these pills are prescribed it is doubted for being oral contraceptives so it is important to have a population- based approach for implementation. Keeping in view the growing deficiencies, a national wheat flour fortification program was developed by health ministry of Pakistan in 2004. This program had aimed to achieve universal flour fortification by the year 2013 [20].

Micronutrient initiative is an organization that is working with Pakistani Government, United nation, and private agencies to achieve certain objectives. One of those objectives is to reduce micronutrient deficiencies in children via the introduction of sprinkles and another is iron interventions for women of childbearing age. Since early 2010, this organization has been working for iron fortification. Special focus is on country's earthquake affected areas. Their objectives include providing assistance in fortification, training mill staff, organizing educational sessions for general public and ensure quality control. They predict that soon all flour produced in Azad Jammu and Kashmir will be fortified. With the help of community organizations, efforts are being generated to strengthen iron and folic acid supplementation program. It is also planned that this organization in collaboration with government will reach out the whole Pakistani population [21].

\section{Intervention plan and impact evaluation}

Realizing the importance and deficiencies of iron, the Government of Pakistan in its 9th 5- year plan has committed for the control of this problem. Studies were done at NIFA (nuclear institute for food and agriculture) Peshawar to test for the feasibility, stability and storage of this product. After establishing a basis for the implementation of this program countrywide, a grant of 3 million US\$ was received from the Global alliance for improved nutrition (GAIN) by the ministry of health. It was predicted that when the project is running at full scale in 2010, 45\% of Pakistan flour will be fortified and 74.2 million people will be consuming this product regularly [22] Pakistan ministry of health gave the contract of manufacturing FeNaEDTA to Fortitech Asia Pacific in 2007 [23].

When micronutrient initiative program was evaluated it was identified that only $11 \%$ of the population is consuming iron-fortified wheat flour and $60 \%$ of women are currently reached by the iron and folic acid supplementation program. The project of iron fortification started in 2007 and it has benefitted half a million people to date [21,23]. In 2008, a study was done to evaluate existing interventions and changes brought by them in maternal and childhood undernutrition. Besides other interventions, it consisted of food supplements and micronutrient interventions. Effective micronutrient intervention in pregnant women consisted of supplementing with ironfolate (improved $\mathrm{Hb} 6.9 \mathrm{~g} / \mathrm{L}$ higher as compared with no fortification). Micronutrient interventions reduced low birth weight at term by $16 \%$. Multiple micronutrients supplements in pregnancy brought $39 \%$ reduction in maternal anemia. This study was designed to examine effects of interventions in 36 countries from Sub -Saharan Africa and South Central Asia. It was concluded that existing interventions could reduce intrauterine growth restrictions, severe wasting and micronutrient deficiencies by $25 \%$. It is thus recommended that these interventions should be continued but work should be done on underlying determinants, that are poverty, poor education, lack of women empowerment and disease burden [24].

Randomized and quasi- experimental trials (31 studies) were also done in both developed and developing countries. It was revealed that daily iron supplement or combination supplement of iron plus folate during pregnancy was associated with $73 \%$ reduction in anemia. According to child health epidemiology reference group guidelines, it is recommended to be used in developing countries [25]. Several interventions exist at the country-wide level and are working effectively but we need to periodically keep on evaluating these interventions in order to identify gaps, accommodate changing needs of populations and make the interventions more effective. There is a need to expand the programs that are already working effectively and efficiently, for the whole population. Work should be done for educating the nation. Empowerment strategies should be implemented especially women empowerment. Resources should be mobilized on an equitable basis. Last but not the least government and private agencies should join together for the benefit of the nation.

\section{Conclusion}

Iron deficiency anemia is one of the important Public Health problems in developing countries among the women of reproductive age group. Multiple factors like age, parity, socioeconomic status and diet can determine the stores of iron in the women of reproductive age group. Anemia can lead to multiple adverse outcomes, which can be prevented by taking appropriate cost-effective interventions on time.

\section{References}

1. Milman N (2011) Anemia-still a major health problem in many parts. Ann Hematol 90: 369-377.

2. Coad J, Conlon C (2011) Iron deficiency in women: assessment, causes and consequences. Curr Opin Clin Nutr Metab Care 14: 625-634.

3. http://web.worldbank.org/archive/website01213/WEB/0_CO-50.HTM

4. Idris M, Rehman AU (2005) Iron deficiency Anemia in moderate to severely anemic patients. J Ayub Med Coll Abbottabad 17: 45-47.

5. Zimmermann MB, Hurrell RF (2007) Nutritional iron deficiency. Lancet 370: 511-520.

6. Siddiqui MS, Siddiqui MK (2008) Public Health significance of iron deficiency anemia. Pak Armed Forces Med J 58: 3.

7. WHO (2008) Global anaemia prevalence and number of individuals affected.

8. WHO (2008) Worldwide prevalence of anaemia 1993-2005.

9. Aspuru K, Villa C, Bermejo F, Herrero P, López SG (2011) Optimal management of iron deficiency anemia due to poor dietary intake. Int J Gen Med 4: 741-750.

10. Stoltzfus RJ (2003) Iron deficiency: Global prevalence and consequences. Food Nutr Bull 24: S99-S103.

11. Milman N (2011) Postpartum anemia I: definition, prevalence, causes, and consequences. Ann Hematol 90: 1247-1253.

12. Lone FW, Qureshi RN, Emmanuel F (2004) Maternal anaemia and its impact on perinatal outcome in a tertiary care hospital in Pakistan. East Mediterr Health J 10: 801-807.

13. Baig-Ansari N, Badruddin SH, Karmaliani R, Harris H, Jehan I, et al. (2008) Anemia prevalence and risk factors in pregnant women in an urban area of Pakistan. Food Nutr Bull 29: 132-139.

14. Cepeda-Lopez AC, Osendarp SJ, Melse-Boonstra A, Aeberli I, GonzalezSalazar F, et al. (2011) Sharply higher rates of iron deficiency in obese Mexican women and children are predicted by obesity-related 
Citation: Mawani M, Ali SA, Bano G, Ali SA (2016) Iron Deficiency Anemia among Women of Reproductive Age, an Important Public Health Problem: Situation Analysis. Reprod Syst Sex Disord 5: 187. doi:10.4172/2161-038X.1000187

Page 6 of 6

inflammation rather than by differences in dietary iron intake. Am J Clin Nutr 93: 975-983.

15. Yanovich R, Merkel D, Israeli E, Evans RK, Erlich T, et al. (2011) Anemia, iron deficiency, and stress fractures in female combatants during 16 months. J Strength Cond Res 25: 3412-3421.

16. Salam RA, Haider BA, Humayun Q, Bhutta ZA (2009) Effect of administration of antihelminthics for soil transmitted helminths during pregnancy. Cochrane Database Syst Rev 18: CD005547.

17. Okwu G, Ukoha A (2008) Studies on the predisposing factors of iron deficiency anaemia among pregnant women in a Nigerian community. Pakistan Journal of nutrition 7: 151-156.

18. Brooker S, Akhwale W, Pullan R, Estambale B, Clarke SE, et al. (2007) Epidemiology of plasmodium-helminth co-infection in Africa: populations at risk, potential impact on anemia, and prospects for combining control. Am J Trop Med Hyg 77: 88-98.

19. Zlotkin SH, Christofides AL, Hyder SM, Schauer CS, Tondeur MC, et al. (2004) Controlling iron deficiency anemia through the use of homefortified complementary foods. Indian J Pediatr 71: 1015-1019.
20. Mahmood F, Zeb A, Khan N (2007) Comparative acceptability studies on roller-milled wheat flour fortified with three different iron fortificants in pakistan. Micronutrient initiative.

21. http://www.micronutrient.org/2009/03/rebuilding-lives-pakistanmicronutrient-fortification/

22. http://www.nifa.org.pk

23. http://www.fortitech.com

24. Bhutta ZA, Ahmed T, Black RE, Cousens S, Dewey K, et al. (2008) What works? Interventions for maternal and child undernutrition and survival. The Lancet 371: 417-440.

25. Yakoob MY, Bhutta ZA (2011) Effect of routine iron supplementation with or without folic acid on anemia during pregnancy. BMC Public Health 11: S21. 